\title{
Effects of an extracellular metal chelator on neurovascular function in diabetic rats
}

\author{
N.E. Cameron, M. A. Cotter \\ Department of Biomedical Sciences, University of Aberdeen, Aberdeen, Scotland, UK
}

\section{Abstract}

Aims/hypothesis. Increased oxidative stress has been causally linked to diabetic neurovascular complications, which are attenuated by antioxidants. There are several possible sources of reactive oxygen species in diabetes. Our aim was to assess the contribution of free radicals, produced by transition metal catalysed reactions, to early neuropathic changes. To this end, we examined, firstly, the effects of an extracellular high molecular weight chelator, hydroxyethyl starch-deferoxamine, which is expected to be confined to vascular space, on nerve perfusion and conduction deficits in diabetic rats and, secondly, the action of a single chelator dose.

Methods. Diabetes was induced by streptozotocin. In vivo measurements comprised sciatic nerve motor conduction velocity and endoneurial perfusion, monitored by hydrogen clearance microelectrode polarography.

Results. We found that 8 weeks of diabetes reduced sciatic blood flow and conduction velocity by $48.3 \%$ and $19.9 \%$ respectively. Two weeks of intravenous treatment corrected these deficits. Starch vehicle was ineffective. The time-course of action of a single hydroxyethyl starch-deferoxamine injection was examined in diabetic rats. There was a rapid increase in nerve blood flow on day 1 , which remained within the non-diabetic range for 9 days before declining to the diabetic level at day 27. In contrast, conduction velocity changes were slower, reaching the non-diabetic range at day 6 and declining to the diabetic level at day 27.

Conclusion/interpretation. Extracellular transition metal catalysed reactions play a major role in the neurovascular deficits of experimental diabetes. Given the long-lasting effect of a single treatment, extracellular metal chelator therapy could be suitable for further assessment in clinical trials. [Diabetologia (2001) 44: 621-628]

Keywords Neuropathy, nerve conduction, blood flow, oxidative stress, iron, copper, chelator, deferoxamine, diabetic rat.
Oxidative stress seems to make an important contribution to the neural and vascular complications of di-

Received: 30 October 2000 and in revised form: 12 January 2001

Corresponding author: Norman E. Cameron, D.Phil., Department of Biomedical Sciences, University of Aberdeen, Institute of Medical Sciences, Foresterhill, Aberdeen AB25 2ZD, Scotland, UK

Abbreviations: AGE, advanced glycation end product; EDRF, endothelium-derived relaxing factor; HES-DFX, hydroxyethyl starch-deferoxamine; NCV, nerve conduction velocity; NO, nitric oxide; ROS, reactive oxygen species. abetes [1, 2]. Experimental models show both impaired nerve antioxidant protection, with decreased glutathione content, and indications of increased lipid peroxidation $[1,3]$. Vascular endothelium function, including endothelium-derived relaxing factor (EDRF) and the nitric oxide (NO) system, is reduced by diabetes-induced oxidative stress [2,4]. Free radical scavengers protect endothelial function [5-7], increase nerve conduction velocity (NCV), blood flow and regenerative capacity [3, 8-12]. The importance of the NO system is emphasised by the fact that antioxidant effects on nerve are eliminated by cotreatment with a NO synthase inhibitor [13]. However, 
high pharmacological doses of scavengers, such as vitamin $\mathrm{E}$, are required to correct neurovascular dysfunction [9]. Thus, an alternative treatment strategy might be to inhibit the production of reactive oxygen species (ROS) rather than simply scavenging them once formed.

Hyperglycaemia enhances ROS production by glucose autoxidation; a reaction catalysed by low concentrations of transition metal ions, iron and copper, which are normally in a bound state but whose homeostasis is compromised by diabetes [14-16]. Glucose autoxidation and some other ROS-producing processes such as the Fenton reaction and autoxidative glycation are inhibited by transition metal chelators [14]. Recently it was shown that deferoxamine (DFX) and trientine treatments improved small and large nerve fibre function, blood flow and endothelium-dependent vasodilation in diabetic rats $[12$, 17-19].

The extent to which adverse ROS effects in diabetic neuropathy are mediated by a direct effect on neuronal metabolic processes and/or or membrane function, or primarily by an indirect effect of a deleterious action on nerve vascular supply is still debated [20]. In experimental diabetic neuropathy, the latter viewpoint is consistent with data from vasodilator studies [21]. Antioxidant treatments that have been examined to date comprise low molecular weight tissue-penetrating compounds, such as vitamin E, lipoic acid, acetyl cysteine or DFX [2, 3, 9-11, 17]. Such agents could have direct neural effects, perhaps overshadowed by their marked vascular actions. In contrast, hydroxyethyl starch-deferoxamine (HES-DFX) is a conjugate of the metal chelator, DFX, and a non-biodegradable high molecular weight dextran. When given intravenously it is largely retained in the vascular space and does not appear to move to other body fluid compartments [22-25]. Treatment with HES-DFX prevented defective NO-mediated endothelium-dependent in the diabetic rat aorta [19]. Thus, the effects and time-course of action of HES-DFX on nerve function and blood flow were examined in diabetic rats to further clarify the role of ROS derived from metal-catalysed reactions and to compare effects with those of tissue-penetrating scavengers.

\section{Methods and materials}

The experiments were performed in accordance with regulations specified by the United Kingdom 'Animal Procedures Act, 1986' and the National Institutes of Health 'Principles of Laboratory Animal Care, 1985 revised version'.

Experimental groups and diabetes induction. Male SpragueDawley rats (Aberdeen University colony) who were 19 weeks old at the start of the study, were used. Diabetes, induced by intraperitoneal streptozotocin (AstraZENECA Pharmaceuticals, Macclesfield, Cheshire, UK) at 40 to $45 \mathrm{mg} / \mathrm{kg}$ freshly dis- solved in sterile $0.9 \%$ saline solution, was verified $24 \mathrm{~h}$ later by estimating hyperglycaemia and glycosuria (Visidex II and Diastix; Ames, Slough, UK). Blood samples were taken from the tail vein for plasma glucose determination (GOD-Perid method; Boehringer Mannheim, Mannheim, Germany) just before final experiments.

The HES-DFX (Biomedical Frontiers, Minneapolis, Minn., USA) is a polydisperse polymer containing DFX, with an average molecular weight of approximately 100000 . It has transition metal chelating properties with a particular affinity for iron, although copper is also strongly bound. It was supplied as an aqueous solution at a polymer concentration of $100 \mathrm{~g} / \mathrm{l}^{-1}$, containing $17 \mathrm{mg} / \mathrm{ml} \mathrm{DFX}$; solution osmolarity was adjusted to $300 \mathrm{mOsm}$ with $\mathrm{NaCl}$. Pharmacokinetic studies of HESDFX show that it is stable in vitro and in vivo; in rats clearance (exclusively renal) is relatively slow, giving a biphasic curve with an initial plasma half life of 4 to $10 \mathrm{~h}$, followed by a prolonged tail so that $10 \%$ of the drug remained in the circulation 4 days after a single intravenous injection [22-24]. Unlike DFX, HES-DFX does not appear to enter cells in vivo, for example, in liver, retina, or optic nerve or cross the blood brain barrier [23, 24, 26, 27]. Experiments in which HES-DFX and DFX were iron loaded to allow histochemical visualisation showed that only the latter entered renal tubular cells cultured in vitro [25].

Two experiments were carried out. In the first, after 6 weeks of untreated diabetes, rats were given twice weekly (Tuesdays and Fridays) injections of $2 \mathrm{ml}$ of HES-DFX solution directly into the tail vein. Final experiments were carried out on day 15 , approximately 3 to 4 days after the last treatment. The treatment regime gave each rat $136 \mathrm{mg}$ DFX over the 15 day period, or approximately $9 \mathrm{mg} /$ day. This was intended to roughly match the effects of daily i.p. injection of native DFX (8 mg), which improved NCV in a previous study [17]. Further, a group of diabetic rats acted as untreated controls, and starch/vehicle controls treated with an equivalent dose ( $2 \mathrm{ml}$ of $100 \mathrm{~g} / \mathrm{l}$ solution) of HES alone using the same regime. The second investigation was designed to examine the timecourse of any effects. A single injection of HES-DFX $(5 \mathrm{ml} /$ $\mathrm{kg}$, resulting in a dose of $85 \mathrm{mg} / \mathrm{kg} \mathrm{DFX}$ ) was given after 6 weeks of untreated diabetes and groups of rats were studied at 1, 3, 6, 9 and 27 day intervals thereafter. A further group of diabetic rats, studied at the day 6 time point, was given an equivalent dose of DFX alone $(85 \mathrm{mg} / \mathrm{kg}$ i.v., dissolved in sterile saline).

Sciatic nerve motor conduction velocity and blood flow. Briefly, under intraperitoneal thiobutabarbital anaesthesia (AstraZENECA; 50-100 mg/kg), the trachea was cannulated for artificial ventilation and a carotid cannula was used to monitor systemic blood pressure. Motor NCV was measured as previously described in the sciatic branch to tibialis anterior muscle [28]. Near-nerve temperature was monitored and kept at $36-37^{\circ} \mathrm{C}$ using radiant heat. Core temperature was regulated between $37-38^{\circ} \mathrm{C}$.

Sciatic endoneurial blood flow was measured by microelectrode polarography and $\mathrm{H}_{2}$ clearance, as previously described [29-31]. Thiobutabarbital anaesthetised rats were given neuromuscular blockade using d-tubocurarine (Sigma, Poole, Dorset, UK); $2 \mathrm{mg} / \mathrm{kg}$ through the carotid cannula) and were artificially ventilated. The level of anaesthesia was monitored by observing any reaction of blood pressure to manipulation and supplementary thiobutabarbital was given as necessary. Body core temperature was maintained at $37-38^{\circ} \mathrm{C}$ and near-nerve temperature was maintained at $35-38^{\circ} \mathrm{C}$ by radiant heat applied to a pre-warmed mineral oil pool that bathed the exposed sciatic nerve. 
Table 1. Body weights and plasma glucose levels

\begin{tabular}{|c|c|c|c|c|}
\hline \multirow[t]{2}{*}{ Group } & \multirow[t]{2}{*}{$n$} & \multicolumn{2}{|c|}{ Weight (g) } & \multirow{2}{*}{$\begin{array}{l}\text { Glucose } \\
\left(\mathrm{mmol} \mathrm{l}^{-1}\right)\end{array}$} \\
\hline & & start & end & \\
\hline Non-diabetic & 10 & $449 \pm 5$ & - & $7.2 \pm 0.6$ \\
\hline Diabetic & 10 & $458 \pm 5$ & $356 \pm 8$ & $39.1 \pm 2.4$ \\
\hline Diabetic + HES & 10 & $475 \pm 8$ & $384 \pm 12$ & $46.2 \pm 1.9$ \\
\hline Diabetic + HES-DFX & 44 & $472 \pm 3$ & $356 \pm 6$ & $40.8 \pm 0.8$ \\
\hline Diabetic + DFX & 6 & $477 \pm 9$ & $383 \pm 12$ & $40.0 \pm 1.9$ \\
\hline
\end{tabular}

Data are means \pm SEM

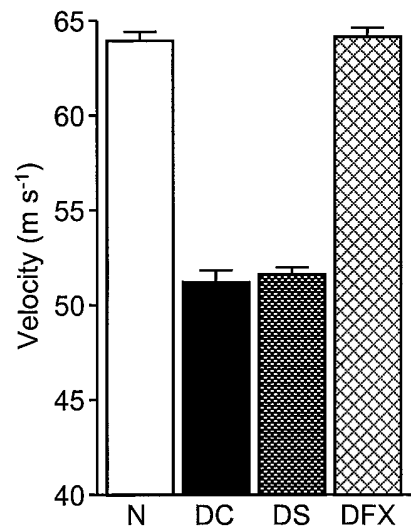

Fig. 1. Effect of diabetes and hydroxyethyl starch deferoxamine treatment on sciatic motor conduction velocity in rats. $\mathrm{N}$, non-diabetic control group $(n=10)$; DC, 8 -week diabetic control group $(n=10)$; DS, 8 -week diabetic group treated for the final 2 weeks with hydroxyethyl starch alone $(n=10)$; DFX, 8 -week diabetic group treated for the final 2 weeks with hydroxyethyl starch deferoxamine $(n=10)$. Data are means + SEM. Statistics: N or DFX vs DC or DS, $p<0.001$; all other comparisons NS

Statistics. Data are expressed as means \pm SEM. They were first subjected to Bartlett's test for homogeneity of variances and were given a log transformation if necessary. One-way ANOVA was then performed, followed by the Bonferroni or Student-Newman-Keuls test to estimate the significance of differences for individual between-group comparisons.

\section{Results}

The diabetic groups of rats had a 5.4 to 6.4-fold increase in plasma glucose (Table 1) compared to nondiabetic control rats. Diabetes of 8 weeks duration caused about a $20 \%$ weight loss in all groups. Plasma glucose and weight loss values were not significantly affected by HES or HES-DFX treatment.

Sciatic motor NCV (Fig. 1) was $19.9 \pm 1.0 \%$ lower $(p<0.001)$ by 8 weeks of diabetes. This was not affected by HES treatment over the final 2 weeks $(19.3 \pm 0.6 \%$ reduction $)$. However, HES-DFX completely corrected $(p<0.001)$ the motor NCV deficit.

Nutritive sciatic endoneurial blood flow (Fig. 2A) was reduced by $48.3 \pm 2.8 \%(p<0.001)$ in the diabet-
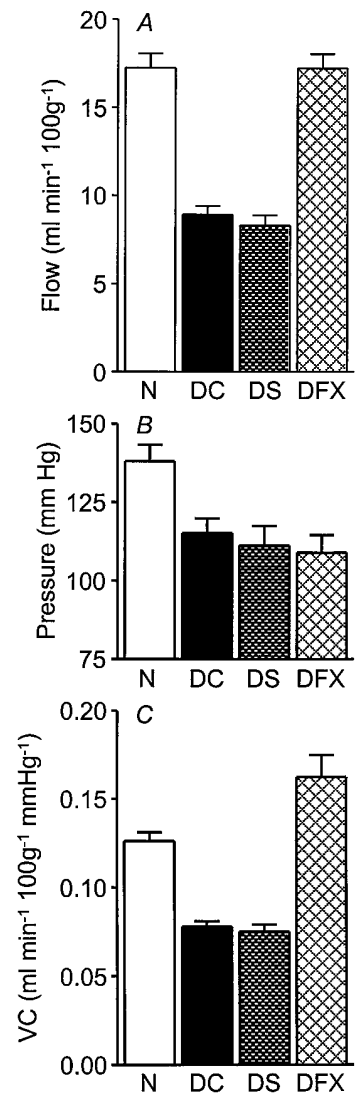

Fig. 2 A-C. Effects of diabetes and hydroxyethyl starch deferoxamine on (A) sciatic endoneurial nutritive blood flow, (B) mean systemic blood pressure, and (C) nutritive vascular conductance in rats. $\mathrm{N}$, non-diabetic control group $(n=10)$; DC, 8 -week diabetic control group $(n=10)$; DS, 8-week diabetic group treated for the final 2 weeks with hydroxyethyl starch alone $(n=10)$; DFX, 8-week diabetic group treated for the final 2 weeks with hydroxyethyl starch deferoxamine $(n=10)$. Data are means + SEM. Statistics: Flow; N or DFX vs DC or DS, $p<0.001$. Pressure; N vs DC, DF or DFX, $p<0.01$. Conductance; N or DFX $v s$ DC or DS, $p<0.001$; N vs DFX, $p<0.01$. All other comparisons NS

ic control group and there was a similar reduction with HES treatment $(51.9 \pm 3.3 \% ; p<0.001)$ compared to the non-diabetic group. The HES-DFX treatment restored blood flow $(p<0.001)$ to within the non-diabetic range. Under the recording conditions, mean systemic blood pressure (Fig. 2B) was $16.5-21.1 \%$ lower in diabetic rats than in non-diabetic rats $(p<0.01)$; there was no significant effect of treatment. Sciatic vasa nervorum does not show appreciable pressure autoregulation. Therefore, to take account of this and obtain a more accurate assessment of treatment effects on vasa nervorum, the perfusion results are also presented as vascular conductance (Fig.2C). This was $38.1 \pm 2.7$ and $40.5 \pm 3.2 \%$ reduced $(p<0.001)$ in diabetic control and HES-treated diabetic groups, respectively. With HES-DFX treatment, conductance was, however, $29.4 \pm 9.5 \%$ supernormal $(p<0.01)$. 

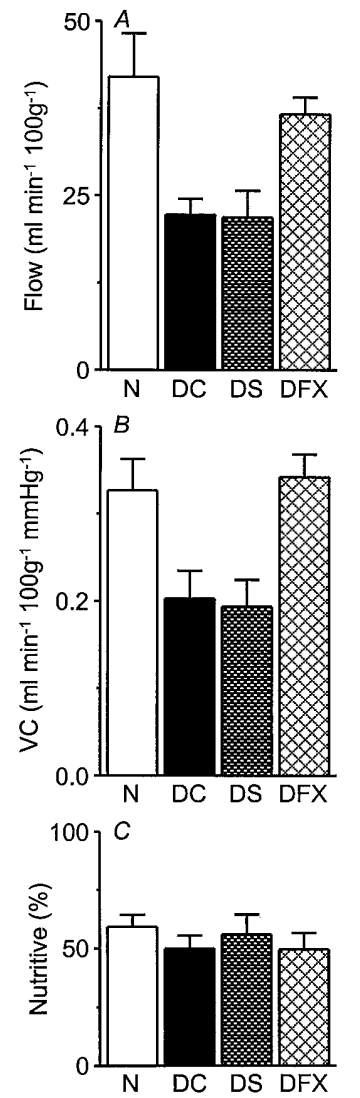

Fig. 3 A-C. Effects of diabetes and hydroxyethyl starch deferoxamine on (A) sciatic total endoneurial blood flow, (B) total vascular conductance, and $(\mathbf{C})$ the proportion of hydrogen clearance carried by the nutritive flow component in rats. $\mathrm{N}$, non-diabetic control group $(n=10)$; DC, 8 -week diabetic control group $(n=10)$; DS, 8 -week diabetic group treated for the final 2 weeks with hydroxyethyl starch alone $(n=10)$; DFX, 8 -week diabetic group treated for the final 2 weeks with hydroxyethyl starch deferoxamine $(n=10)$. Data are means + SEM. Statistics: Flow; N vs DC or DS, $p<0.001$; DFX vs DC or DS, $p<0.01$. Conductance; $\mathrm{N} v s$ DC or DS, $p<0.05$; DFX $v s \mathrm{DC}$ or DS, $p<0.01$. All other comparisons NS

Total sciatic endoneurial flow (Fig. 3A) was also reduced by $47.0 \pm 5.5 \%$ and $48.0 \pm 9.2 \%(p<0.001)$ in diabetic control groups and HES-treated diabetic groups respectively. This was $73.2 \pm 12.2 \%$ corrected $(p<0.01)$ by HES-DFX treatment. When analysed in terms of vascular conductance (Fig.3B) the 39\% diabetic deficit $(p<0.01)$ was corrected completely by HES-DFX $(p<0.01)$, the resultant value being in the upper half of the non-diabetic range. There was no effect of diabetes or treatment on the pattern of endoneurial blood flow reflected as the percentage of hydrogen clearance carried by the nutritive component (Fig. 3C).

The time-course of action of a single intravenous injection of HES-DFX in diabetic rats is shown in Figure 4. Within 1 day of HES-DFX treatment, sciatic nutritive blood flow increased to the non-diabetic range $(p<0.001)$, followed by a decline to the diabet-

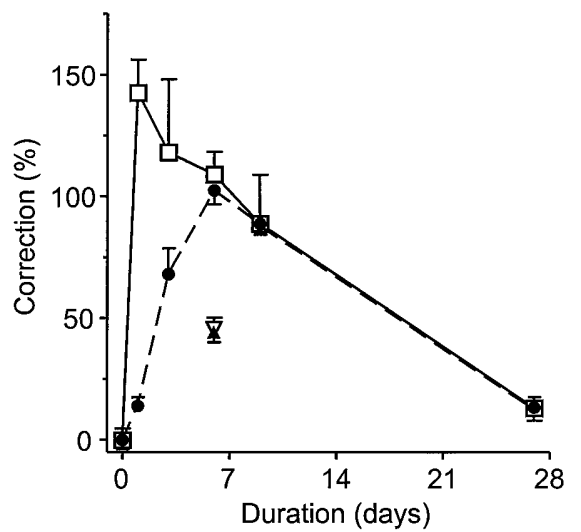

Fig.4. Time-course of effects of a single dose of hydroxyethyl starch-deferoxamine on sciatic motor conduction velocity (dashed line, 0 ) and nutritive blood flow (solid line, $\square$ ). Data are means \pm SEM $(n=6-8)$ and are expressed as percentage correction of the diabetic deficit at 1,3,6, 9, and 27 days posttreatment to facilitate comparison between conduction velocity and blood flow. Also shown are data (blood flow, conduction velocity, $\boldsymbol{\Delta}$ ) at the 6-day time-point for a group of diabetic rats $(n=6)$ treated with DFX alone. Blood flow was improved by HES-DFX $(p<0.001)$ compared to the untreated diabetic level in 1,3,6, and 9 day groups and did not differ from the non-diabetic level except at 27 days $(p<0.001)$. Conduction velocity was not significantly increased at day 1 , partially improved at day 3 ( $p<0.001 v s$ non-diabetic and diabetic levels), normalised at day $6(p<0.001)$, with a return to diabetic level at day $27(p<0.001)$. Treatment with DFX alone partially corrected conduction velocity and blood flow after 6 days ( $p<0.01$ vs. non-diabetic and diabetic levels), the effect being less than for HES-DFX $(p<0.01)$

ic range after 27 days. Values up to 9 days were not significantly different from the non-diabetic range and were higher $(p<0.001)$ than in untreated diabetes. In marked contrast, motor NCV effects lagged behind those for blood flow. The NCV was not significantly altered 1 day after treatment; there was partial correction $(68.1 \pm 10.6 \%, p<0.001)$ after 3 days and complete correction after 6 days. Following that, $\mathrm{NCV}$ declined at a rate closely matching that for blood flow, reaching a value within the diabetic range after 27 days. In diabetic rats given a single injection of DFX alone and studied 6 days later, the magnitudes of correction of the NCV and blood flow deficits were similar $(44.2 \pm 4.1 \%$ and $45.4 \pm 4.7 \%$, respectively; $p<0.001)$.

\section{Discussion}

Treatment with HES-DFX corrected sciatic motor NCV and blood flow deficits in diabetic rats, whereas HES alone was without an effect: the DFX moiety was therefore the active agent. This agrees with and extends the conclusions from previous transition metal chelator studies. Thus, DFX restored motor and sensory NCV, sciatic nerve blood flow and vasa ner- 
vorum endothelium-dependent relaxation [17, 32] and HES-DFX protected against the development of aorta NO-mediated endothelium-dependent relaxation deficits [19] in diabetic rats. Furthermore, trientine corrected NCV deficits, improved regenerative capacity in diabetic and galactosaemic rats $[12,17]$, increased nerve perfusion, protected EDRF in aorta and corpus cavernosum and improved the action of cavernosal nitrergic innervation $[17,18]$. Previous experiments showed that DFX or trientine had no effect on NCV or nerve blood flow in non-diabetic rats, emphasising the importance of impaired transition metal homeostasis in diabetes [15-17]. Taken together and given the substantial beneficial effects of chelator treatment, the data provide strong support for the hypothesis that transition metal catalysed reactions play a major role in the development of neurovascular dysfunction in experimental diabetes.

In a preventative study of chronic HES-DFX effects on aorta [19] from diabetic rats there was a reduction in glycated haemoglobin, perhaps indicating that improved metabolic control played a part in HES-DFX's vascular action. While glycated haemoglobin was not assessed in this study, other sensitive indicators such as plasma glucose and body weight did not show any evidence of improved metabolic control. With intensive insulin treatment, near normoglycaemic conditions are necessary to correct nerve dysfunction in diabetic rats [33], therefore it is not likely that any subtle putative action of HESDFX on metabolic control contributed appreciably to the present results.

While the experiments do not elucidate the precise details of the transition metal catalysed reactions involved, there are several candidates. Glucose autoxidation is a potentially important mechanism [14], producing both ROS and reactive carbonyl compounds such as glyoxal that participate in advanced glycation reactions $[34,35]$. In turn, superoxide reacts with NO to produce peroxynitrite, which impairs vasodilation, and can nitrosylate proteins to alter their function [36]. Decomposition of peroxynitrite liberates the cytotoxic hydroxyl radical and transition metals catalyse the production of hydroxyl radicals from superoxide by the Fenton reaction. The importance of hydroxyl radicals for impaired endothelial function in diabetes has been stressed [37]. Moreover, the complex process of advanced glycation end product (AGE) formation produces ROS by metal-catalysed reactions, and AGEs themselves can neutralise NO [38]. Recent studies have also shown that AGEs bind transition metals, resulting in a greatly increased potential for degradation of NO-containing EDRFs $[39,40]$. Furthermore, increases in cellular transition metals, particularly copper, cause a decline in mitochondrial membrane potential, accompanied by ROS formation [41]. Thus, compelling biochemical arguments predict marked neurovascular conse- quences of transition metal chelator treatment in diabetes.

Treatment with HES-DFX completely reversed the sciatic nutritive blood flow deficit in diabetic rats; vascular conductance was supernormal which emphasises its effectiveness. Furthermore, total endoneurial perfusion, comprising nutritive, large vessel and ateriovenous shunt flow, was completely corrected by HES-DFX. This indicates a general effect on vasa nervorum control mechanisms without changes in flow pattern. This is in accord with the effects of ROS scavengers such as vitamin E [9], probucol [8], or lipoic acid which has both scavenger and chelator properties [42]. In contrast, the sulphydryl donor, acetyl cysteine [10], the AGE inhibitor aminoguanidine [43], or aldose reductase inhibitors [44] had more specific actions favouring nutritive flow.

Experiments on the time-course of effects of a single high dose of HES-DFX, showed a very rapid action on nutritive blood flow, occurring within one day of intravenous administration. This suggests that the vasa nervorum deficits after 8 weeks of diabetes are likely to depend more on biochemical than structural defects, a likely scenario being interference by transition metal catalysed ROS production of vasodilator (NO) function [32] which is known to be compromised by diabetes $[45,46]$. Experiments on NOmediated vasodilation of aorta from diabetic rats showed that acute in vitro exposure to the metal chelator, trientine, did not correct impaired responses whereas chronic in vivo treatment prevented dysfunction [18]. If the mechanism of vasa nervorum dysfunction has parallels with that of aorta, then it is plausible that blood itself is an important source of transition metals that impair nerve perfusion. This argument is reinforced by the polymeric nature of HES-DFX, restricting distribution to the vascular body fluid compartment. Thus, HES-DFX would act as a "sink" for circulating free transition metals, chelating them to reduce ROS formation, with eventual elimination by renal clearance. This does not preclude potential effects due to the uptake of transition metals from other extravascular sites by mass action. Data from in vitro aorta experiments [18] suggest, however, that one postulated site of transition metal accumulation, bound to AGEs in subendothelial collagen, is relatively unimportant for vascular dysfunction.

In contrast to blood flow, recovery of NCV after a single HES-DFX treatment was delayed by up to 6 days. This is compatible with a causal relationship on a vascular hypothesis of diabetic neuropathy. A similar delay (6-7 days) between blood flow and NCV correction has been observed for treatment with vasodilators (angiotensin II antagonists) and vasodilator substrates $\omega$-6 essential fatty acids) [47-49]. The reason for the diabetic NCV deficit is not clear. It is not likely to depend on nerve fibre damage; mor- 
phological changes are subtle after only 8 weeks of streptozotocin-induced diabetes [50, 51]. Rather, reduced excitability and NCV could result from $\mathrm{Na}^{+}$ channel inactivation due to chronic depolarisation in diabetic rat nerves [52]; similar changes occur when non-diabetic nerves are exposed to anoxia [53]. The depolarisation could result from an adaptation to reduced energy supply for $\mathrm{Na}^{+}, \mathrm{K}^{+}$ATPase pumps [54]. Correction of the nerve perfusion deficit would improve ATP production [20], restoring membrane potential and NCV. This differs from the situation found in chronic clinical neuropathy, where there is substantial nerve fibre damage that needs to be repaired for maximum NCV restoration [55]. However, parallels could exist with the relatively rapid NCV improvement seen in clinical trials of the potent aldose reductase inhibitor, minalrestat, presumably reflecting return of function in a subset of undamaged fibres [56].

Perhaps the most striking aspect was the longevity of effects of a single HES-DFX treatment; which brought blood flow within the non-diabetic range for 9 days, allowing the complete correction of NCV. Although HES-DFX has a relatively long plasma halflife, this is not sufficient to explain the effect. Furthermore, in other experiments which studied the washout of drug effects, NCV returned rapidly to the diabetic level following cessation of treatment (2-4 days for lisinopril, $\gamma$-linolenic acid, the antioxidant, probucol, and the aldose reductase inhibitor, minalrestat $[8,47,49,57])$. A possible explanation is that the long duration of HES-DFX action relates to the removal of free transition metals from the body and that a substantial time is necessary for levels to build up again to those of untreated diabetes.

The effect of a single injection of HES-DFX was compared with that of an equivalent dose of DFX. The latter produced about half the functional effect of the former when tested 6 days later. Compared with HES-DFX, DFX has a short plasma half-life, being rapidly eliminated by the kidneys, although it does move to interstitial space. It is possible that this duration was not sufficient for efficient chelation of all available transition metals after a single dose, although repeated daily treatment fully restores NCV and nerve perfusion [17]. The reduced action of DFX compared with HES-DFX shows that any dissociation of the latter in vivo, to liberate free DFX, would not be expected to contribute positively to the marked neurovascular effects.

In conclusion, the profound action of transition metal chelators on nerve function and perfusion in diabetic rats, coupled with the longevity of the effects of a single treatment, suggest that this approach might be suitable for study in clinical neuropathy trials. Despite the need for intravenous HES-DFX injection, this drug has two potential advantages. First, injection might only be needed on a monthly basis.
Second, DFX penetrates the interstitium and cells of the retina and prolonged treatment can cause retinopathy but HES-DFX is without these adverse side effects [23].

Acknowledgements. This work was supported in part by Juvenile Diabetes Foundation International grant \#1-2000-45. We are grateful to Biomedical Frontiers Inc for the gifts of HESDFX and HES.

\section{References}

1. Low PA, Nickander KK (1991) Oxygen free radical effects in sciatic nerve in experimental diabetes. Diabetes 40: 873-877

2. Cameron NE, Cotter MA (1999) Effects of antioxidants on nerve and vascular dysfunction in experimental diabetes. Diabetes Res Clin Pract 45: 137-146

3. Nagamatsu M, Nickander KK, Schmelzer JD et al. (1995) Lipoic acid improves nerve blood flow, reduces oxidative stress and improves distal nerve conduction in experimental diabetic neuropathy. Diabetes Care 18: 1160-1167

4. Pieper GM (1998) Review of alterations in endothelial nitric oxide production in diabetes: protective role of arginine on endothelial dysfunction. Hypertension 31: 1047-1060

5. Keegan A, Walbank H, Cotter MA, Cameron NE (1995) Chronic vitamin $\mathrm{E}$ treatment prevents defective endothelium-dependent relaxation in diabetic rat aorta. Diabetologia 38: 1475-1478

6. Archibald V, Cotter MA, Keegan A, Cameron NE (1996) Contraction and relaxation of aortas from diabetic rats: effects of chronic anti-oxidant and aminoguanidine treatments. Naunyn-Schmeidebergs Arch Pharmacol 353: 584-591

7. Rösen P, Ballhausen T, Bloch W, Addicks K (1995) Endothelial relaxation is disturbed by oxidative stress in the diabetic rat heart: influence of tocopherol as antioxidant. Diabetologia 38: 1157-1168

8. Cameron NE, Cotter MA, Archibald V, Dines KC, Maxfield EK (1994) Anti-oxidant and pro-oxidant effects on nerve conduction velocity, endoneurial blood flow and oxygen tension in non-diabetic and streptozotocin-diabetic rats. Diabetologia 37: 449-459

9. Cotter MA, Love A, Watt MJ, Cameron NE, Dines KC (1995) Effects of natural free radical scavengers on peripheral nerve and neurovascular function in diabetic rats. Diabetologia 38: 1285-1294

10. Love A, Cotter MA, Cameron NE (1996) Effects of the sulphydryl donor $N$-acetyl-1-cysteine on nerve conduction, perfusion, maturation and regeneration following freeze damage in diabetic rats. Eur J Clin Invest 26: 698-706

11. Sagara M, Satoh J, Wada R et al. (1996) Inhibition of development of peripheral neuropathy in streptozotocin-induced diabetic rats with n-acetylcysteine. Diabetologia 39: 263-269

12. Love A, Cotter MA, Cameron NE (1996) Nerve function and regeneration in diabetic and galactosaemic rats: antioxidant and metal chelator effects. Eur J Pharmacol 314: 33-39

13. Cameron NE, Cotter MA (1995) Reversal of peripheral nerve conduction and perfusion deficits by the free radical scavenger, BM15.0639, in diabetic rats. Naunyn-Schmiedebergs Arch Pharmacol 352: 685-690 
14. Wolff SP (1995) Diabetes mellitus and free radicals. $\mathrm{Br}$ Med Bull 49: 642-652

15. Failla ML, Kiser RA (1981) Altered tissue content and cytosol distribution of trace metals in experimental diabetes. J Nutr 111: 1900-1909

16. Walter RM, Uriu-Hare JY, Olin KL et al. (1991) Copper, zinc, manganese, and magnesium status and complications of diabetes mellitus. Diabetes Care 14: 1050-1056

17. Cameron NE, Cotter MA (1995) Neurovascular deficits in diabetic rats: potential contribution of autoxidation and free radicals examined using transition metal chelating agents J Clin Invest 96: 1159-1163

18. Keegan A, Cotter MA, Cameron NE (1999) Effects of chelator treatment on aorta and corpus cavernosum from diabetic rats. Free Radic Biol Med 27: 536-543

19. Pieper GM, Siebeneich W (1997) Diabetes-induced endothelial dysfunction is prevented by long term treatment with the modified iron chelator, hydroxyethyl starch conjugated-deferoxamine. J Cardiovasc Pharmacol 30: 734-738

20. Obrosova IG, Van Huysen C, Fathallah L, Cao X, Stevens M, Greene D (2000) Evaluation of $\alpha_{1}$-adrenoceptor antagonist on diabetes-induced changes in peripheral nerve function, metabolism, and antioxidative defense. FASEB J 14: $1548-1558$

21. Cameron NE, Cotter MA (2000) New therapeutic approaches to prevent or halt the progression of the longterm complications of diabetes mellitus: focus on neuropathy. Curr Opin Oncol 2: 162-177

22. Hallaway PE, Eaton JW, Panter SS, Hedlund BE (1989) Modulation of deferoxamine toxicity and clearance by covalent attachment to biocompatible polymers. Proc Natl Acad Sci USA 86: 10108-10112

23. Gehlbach PL, Purple RL, Hallaway PE, Hedlund BE (1993) Polymer conjugation reduces deferoxamine induced retinopathy in an albino rat model. Invest Ophthalmol Vis Sci 34: 2871-2877

24. Moch D, Schroppel B, Schoenberg MH et al. (1995) Protective effects of hydroxyethyl starch-deferoxamine in early sepsis. Shock 4: 425-432

25. Paller MS, Hedlund BE (1994) Extracellular iron chelators protect kidney cells from hypoxia/reoxygenation. Free Radic Biol Med 17: 597-603

26. Guy J, McGorray S, Qi, X, Fitzsimmons J, Mancusco A, Rao N (1994) Conjugated deferoxamine reduces bloodbrain barrier disruption in experimental optic neuritis. Opthalmic Res 26: 310-323

27. Sadrzadeh SM, Hallaway PE, Nanji AA (1997) The longacting parenteral iron chelator, hydroxyethyl starch-deferoxamine, fails to protect against alcohol-induced liver injury in rats. J Pharmacol Exp Ther 280: 1038-1042

28. Cameron NE, Cotter MA, Robertson S (1989) The effect of aldose reductase inhibition on the pattern of nerve conduction deficits in diabetic rats. Q J Exp Physiol 74: 917-926

29. Cameron NE, Cotter MA, Hohman TC (1996) Interactions between essential fatty acid, prostanoid, polyol pathway and nitric oxide mechanisms in the neurovascular deficit of diabetic rats. Diabetologia 39: 172-182

30. Cameron NE, Cotter MA, Basso M, Hohman TC (1997) Comparison of the effects of inhibitors of aldose reductase and sorbitol dehydrogenase on neurovascular function, nerve conduction and tissue polyol pathway metabolites in streptozotocin-diabetic rats. Diabetologia 40: 271-281

31. Low PA, Lagerlund TD, McManis PG (1989) Nerve blood flow and oxygen delivery in normal, diabetic and ischemic neuropathy. Int Rev Neurobiol 31: 355-438
32. Cameron NE, Cotter MA (2000) Antioxidants in the treatment of diabetic polyneuropathy: synergy with essential fatty acids. In: (eds) Packer L, Rosen P, King G, Azzi A, Tritschler H Oxidative Stress and Diabetes Management Decker, New York, pp 129-154

33. Greene DA, De Jesus PV, Winegrad AI (1975) Effects of insulin and dietary myoinositol on impaired peripheral motor nerve conduction velocity in acute streptozotocin diabetes. J Clin Invest 55: 1326-1336

34. Baynes JW, Thorpe SR (1999) Role of oxidative stress in diabetic complications: a new perspective on an old paradigm. Diabetes 48: 1-9

35. Thornally PJ, Langborg A, Minhas HS (1999) Formation of glyoxal, methylglyoxal, and 3-deoxyglucasone in the glycation of protein by glucose. Biochem J 344: 109-116

36. Beckman JS, Beckman TW, Chen J, Marshall PA, Freeman BA (1990) Apparent hydroxyl radical production by peroxynitrite: implications for endothelial injury from nitric oxide and superoxide. Proc Natl Acad Sci USA 87: 1620-1624

37. Pieper GM, Siebeneich W, Roza AM, Jordan M, Adams MB (1996) Chronic treatment in vivo with dimethylthiourea, a hydroxyl radical scavenger, prevents diabetes-induced endothelial dysfunction. J Cardiovasc Pharmacol 28: 741-745

38. Bucala R, Tracey K, Cerami A (1991) Advanced glycosylation products quench nitric oxide and mediate defective endothelium-dependent vasodilation in experimental diabetes. J Clin Invest 87: 432-438

39. Qian M, Liu M, Eaton JM (1998) Transition metals bind to glycated proteins forming redox active "glycochelates": Implications for the pathogenesis of certain diabetic complications. Biochem Biophys Res Commun 250: 385-389

40. Saxena AK, Saxena P, Wu X, Obrenovich M, Weiss MF, Monnier VM (1999) Protein aging by carboxymethylation of lysines generates sites for divalent metal and redox active copper binding: Relevance to diseases of glycoxidative stress. Biochem Biophys Res Commun 260: 332-338

41. Pourahmad J, O'Brien PJ (2000) A comparison of hepatocyte cytotoxic mechanisms for $\mathrm{Cu}^{2+}$ and $\mathrm{Cd}^{2+}$. Toxicology 143: 263-273

42. Cameron NE, Cotter MA, Horrobin DH, Tritschler HJ (1998) Effects of $\alpha$-lipoic acid on neurovascular function in diabetic rats: interaction with essential fatty acids. Diabetologia 41: 390-399

43. Cameron NE, Cotter MA (1996) Rapid reversal by aminoguanidine of the neurovascular effects of diabetes in rats: modulation by nitric oxide synthase inhibition. Metabolism 45: $1147-1152$

44. Cameron NE, Cotter MA, Dines KC, Maxfield EK, Carey F, Mirrlees DJ (1994) Aldose reductase inhibition, nerve perfusion, oxygenation and function in streptozotocin-diabetic rats: dose-response considerations and independence from a myo-inositol mechanism. Diabetologia 37: 651-663

45. Kihara M, Low PA (1995) Impaired vasoreactivity to nitric oxide in experimental diabetic neuropathy. Exp Neurol 132: $180-185$

46. Maxfield EK, Cameron NE, Cotter MA (1997) Effects of diabetes on reactivity of sciatic vasa nervorum in rats. J Diabetes Complications 11: 47-55

47. Cameron NE, Cotter MA, Robertson S (1993) Rapid reversal of a motor nerve conduction deficit in streptozotocin-diabetic rats by the angiotensin converting enzyme inhibitor lisinopril. Acta Diabetol 30: 46-48

48. Maxfield EK, Cameron NE, Cotter MA, Dines KC (1993) Angiotensin II receptor blockade improves nerve function, modulates nerve blood flow and stimulates angiogenesis in streptozotocin-diabetic rats. Diabetologia 36: 1230-1237 
49. Dines KC, Cotter MA, Cameron NE (1995) Comparison of the effects of evening primrose oil and triglycerides containing $\gamma$-linolenic acid on nerve conduction and blood flow in diabetic rats. J Pharmacol Exp Ther 273: 49-55

50. Cameron NE, Leonard MB, Ross IS, Whiting PH (1986) The effects of sorbinil on peripheral nerve conduction velocity, polyol concentrations and morphology in the streptozotocin-diabetic rat. Diabetologia 29: 168-174

51. Yagihashi S (1995) Pathology and pathogenetic mechanisms of diabetic neuropathy. Diabetes Metab Rev 11: 193-225

52. Brismar T, Sima AAF (1981) Changes in nodal function in nerve fibres of the spontaneously diabetic BB-Wistar rat: potential clamp analysis. Acta Physiol Scand 113: 499-506
53. Brismar T (1981) Potential clamp analysis of the effect of anoxia on the nodal function of rat peripheral nerve fibres. Acta Physiol Scand 112: 495-496

54. Ritchie JM (1985) A note on the mechanism of resistance to anoxia and ischaemia in the pathophysiological mammalian myelinated nerve. J Neurol Neurosurg Psychiatry 48: 274-277

55. Tesfaye S, Malik R, Ward JD (1994) Vascular factors in diabetic neuropathy. Diabetologia 37: 847-854

56. Efanova I (1999) Minalrestat. Curr Opin Oncol 1: 101-107

57. Basso M, Banas D, Lai K-D, Hohman TC, Cameron NE, Cotter MA (1998) Oxidative stress and Na/K ATPase activity in diabetic rats: aldose reductase inhibitor withdrawal studies. Diabetologia 41 [Suppl 1]: A271 (Abstract) 